\title{
On the linear stability of some finite difference schemes for nonlinear reaction-diffusion models of chemical reaction networks
}

\author{
Nathan Muyinda $^{1 *}$, Bernard De Baets ${ }^{2}$, Shodhan Rao $^{1}$ \\ ${ }^{1}$ Ghent University Global campus, 119 Songdomunhwa-Ro, Yeonsu-Gu, Incheon, South Korea \\ ${ }^{2}$ KERMIT, Department of Data Analysis and Mathematical Modelling, Ghent University, Coupure links 653, \\ B-9000 Gent, Belgium \\ *Email address for correspondence: Nathan.Muyinda@Ugent.be \\ Communicated by Luca Formaggia \\ Received on 10 03, 2017. Accepted on 10 08, 2018.
}

\begin{abstract}
We identify sufficient conditions for the stability of some well-known finite difference schemes for the solution of the multivariable reaction-diffusion equations that model chemical reaction networks. Since the equations are mainly nonlinear, these conditions are obtained through local linearization. A recurrent condition is that the Jacobian matrix of the reaction part evaluated at some positive unknown solution is either D-semi-stable or semi-stable. We demonstrate that for a single reversible chemical reaction whose kinetics are monotone, the Jacobian matrix is D-semi-stable and therefore such schemes are guaranteed to work well.

Keywords: Reaction-diffusion equations, Finite difference method, Stability, Amplification matrix, Linearization, Monotone kinetics.

AMS subject classification: 35K57, 65M06, 65M12, 80A30, 92C45
\end{abstract}

\section{Introduction}

Reaction-diffusion (RD) equations are mathematical models that have proven to be a powerful tool for describing a broad class of phenomena in physics, chemistry and biology. These equations describe how the time evolution of the concentration density of species in space varies under the influence of two processes; local interaction of species and diffusion which causes the spread of species in space.

R.A. Fisher in 1937 [1] was the first to propose a reaction-diffusion equation, which is now called the classic Fisher equation, as a model for the spread of a new recessive advantageous gene through a population. Since then RD equations have found applications in the field of population genetics (e.g. [2,3]). J.G. Skellam in 1951, in his pioneering paper [4] used a similar framework for theoretical studies of population dispersal and introduced the RD equation in ecology. The seminal paper of A. Turing in 1952 [5] demonstrated how a simple model system of two coupled RD equations could be used as a model for biological pattern formation via a mechanism known as diffusion-driven instability. Turing's theory sparked a wide range of research in morphogenesis, notable among which the works of Gierer and Meinhardt [6] and Murray $[7,8]$. RD equations have also been applied in the study of spatio-temporal patterns produced by the Belousov-Zhabotinsky chemical reaction [9], in the modelling of calcium dynamics [10-14] and in the theory of wave propagation in excitable media [15], among others.

The simplest RD model can be described by an equation

$$
\frac{\partial \mathbf{u}}{\partial t}=D \nabla^{2} \mathbf{u}+\mathbf{f}(\mathbf{u})
$$

where $\mathbf{u}$ is a vector of species concentration densities, $D$ is a constant matrix of diffusion coefficients which, if there is no cross-diffusion among the species, is simply a positive diagonal matrix, $\nabla^{2}$ is the Laplacian, and $\mathbf{f}(\mathbf{u})$ is a vector of functions representing reaction kinetics. The problem is fully specified once the appropriate initial conditions $\mathbf{u}(\mathbf{x}, 0)=\mathbf{u}_{0}(\mathbf{x})$ and boundary conditions are known. 
In many problems, the reaction term $\mathbf{f}(\mathbf{u})$ is mainly nonlinear, arising, for example, from the use of the law of mass action or Michaelis-Menten kinetics which makes Equation (1) nonlinear (also called semilinear). This nonlinearity makes it, in most cases, very difficult (if not impossible) to solve the equation analytically and justifies the frequent use of numerical methods such as finite difference schemes.

A usual first step in the application of numerical methods is the use of the method of lines (MOL) [16] which reduces the RD system to a semi-discrete form, consisting of nonlinear ordinary differential equations (ODEs)

$$
\mathbf{u}_{t}=\mathcal{C} \mathbf{u}+\mathcal{F}(\mathbf{u})
$$

Here $\mathcal{C}$ is a constant matrix arising out of the discretization of the Laplacian plus boundary conditions and $\mathcal{F}(\mathbf{u})$ is a vector of the nonlinear reaction terms evaluated at the grid points. An ODE integration technique can now be used to integrate the problem. However, there are two drawbacks about this ODE system:

- the dimension of the system is huge, especially if the partial differential equation (PDE) in Equation (1) is in three spatial dimensions and for reaction networks involving many reacting species. This puts a constraint on the use of standard implicit difference schemes since, at every time step, an iterative solver like Newton's method or Picard iteration has to be employed to solve a nonlinear system of equations.

- the system is stiff. There is no precise mathematical definition for stiffness. However, the notion of stiffness is characterized by the appearance of different time scales in the solutions [17]. Stiffness commonly arises due to the approximation of the diffusion term by finite differences, but it is also prevalent in RD equations for reaction networks which often involve different chemical reactions taking place on vastly differing time scales. The reactions can vary in time scales from microseconds to days due to large reaction constants. Thus, in order to ensure stability of the easyto-use explicit schemes, one will be restricted to using very small time steps that are determined by not only the Courant-Friedrichs-Lewy (CFL) condition but also by the smallest time constant. However, the time for all chemical species to reach near equilibrium values depends on the largest time constant. As a result, these methods may require excessive amounts of computer time to solve stiff systems of ODEs [18].

A number of researchers propose the use of implicit schemes, e.g. the Crank-Nicolson (CN) scheme [19] and fractional step $\theta$-scheme (FSTS) [20,21], given the fact that such schemes have already been proven to be unconditionally stable for the linear diffusion equation without the reaction term. The belief is that this unconditional stability extends to the full RD equation with the (in general) nonlinear reaction term, although, as far as we know, this has not been explicitly established. The main drawback of implicit schemes, especially for nonlinear RD systems, is the computational complexity in their implementation. In this regard, a number of researchers have also devoted time to proposing explicit schemes that are efficient enough to match the implicit schemes, e.g. the exponential time-differencing fourth order Runge-Kutta (ETDRK) [22], Implicit-Explicit (IMEX) schemes [23,24], which approximate the linear diffusion terms implicitly and the nonlinear reaction terms explicitly. Others have come up with semi-implicit schemes that are linearly unconditionally stable and may be easier to implement than the fully implicit schemes. Examples include the second-order semi-implicit backward differentiation formula (2-SBDF) [25] and the implicit integration factor (IIF) method [26,27].

In this paper, we establish sufficient conditions for the stability of some popular finite difference schemes for the nonlinear multivariable RD system (1). The motivation for this is the fact that stability plays a major role in deciding which numerical method to use for the solution of Equation (1) and yet not many studies have been devoted to such a stability analysis. Many stability conditions for finite difference schemes in the literature have been established based on an analysis of scalar equations or in the case of systems, the linear diffusion equation (see e.g. [28]). One notable paper on stability of finite difference schemes for the nonlinear RD equations is by Hoff [29]. However, Hoff's analysis makes use of 
deep theoretical concepts from functional analysis which may not be embraced by many users of finite difference schemes for practical purposes. Other related work can be found in e.g. [30,31]. The methods used in this paper are similar to the method used by Li et al. [32] to analyze the stability of an explicit finite difference method applied to a scalar nonlinear RD equation in two spatial dimensions. However, chemical reaction networks involving only a single chemical species are hard to find in practice. Most chemical reaction networks involve at least two chemical species and thus the RD models of such networks are multivariable. We thus believe that from a practical point of view, there is still a need for a rigorous study of stability of finite difference schemes, particularly for nonlinear RD equations in the multivariable case. This paper is therefore aimed at contributing towards this cause.

The paper is organized as follows. A linearization of Equation (1) is performed in Section 2. Some mathematical concepts and theorems are given in Section 3. In Section 4, a local stability analysis of some popular difference schemes applied to the linearized system is carried out. We show in Section 5 that for RD systems whose reactions are governed by a variety of enzyme kinetic rate laws, some of the established sufficient conditions are naturally satisfied implying that these implicit schemes are expected to work for the solution of such RD systems. In Section 6, we perform numerical experiments on the Brusselator RD system and finally present our conclusions in Section 7.

\section{Linearization}

Investigating stability of a numerical scheme for a nonlinear problem like (1) can be a complicated task because the exact analytical solution is not known. For this reason, we investigate stability of difference schemes for the localized linear version. It should however be noted that although the linearization substantially simplifies the stability analysis, the conclusions of a linear stability analysis may not necessarily apply to the nonlinear problem. The stability analysis of the locally linearized system applies approximately and locally. This is due to the fact that linear stabilty is a necessary condition for nonlinear problems but is certainly not sufficient [33].

To carry out a linearization, suppose $\mathbf{u}$ and $\mathbf{v}$ are two analytical solutions to Equation (1) that are close to each other. Then their difference satisfies

$$
\frac{\partial}{\partial t}(\mathbf{u}-\mathbf{v})=D \nabla^{2}(\mathbf{u}-\mathbf{v})+\mathbf{f}(\mathbf{u})-\mathbf{f}(\mathbf{v})
$$

Define $\mathbf{z}:=\mathbf{u}-\mathbf{v}$. A Taylor expansion of $\mathbf{f}(\mathbf{u})$ about $\mathbf{v}$ leads to

$$
\frac{\partial \mathbf{z}}{\partial t}=D \nabla^{2} \mathbf{z}+A \mathbf{z}+\mathcal{O}\left(\mathbf{z}^{2}\right)
$$

where $A$ is the Jacobian matrix of $\mathbf{f}$ evaluated at $\mathbf{v}$, and so at any instant in space and time, it is a matrix of constants. When $\mathbf{z}$ is small, which corresponds to the two solutions $\mathbf{u}$ and $\mathbf{v}$ being near each other, such that the $\mathcal{O}\left(\mathbf{z}^{2}\right)$ terms are small compared to the linear term $A \mathbf{z}$, the difference between the two solutions satisfies:

$$
\frac{\partial \mathbf{z}}{\partial t} \approx D \nabla^{2} \mathbf{z}+A \mathbf{z}
$$

Equation (3) is the local linear approximation (or linearization) of Equation (1) about the solution v. In all the analyses of this paper, we assume that the Jacobian $A$ is real with finite entries in the space and time domains under consideration.

\section{Some mathematical concepts}

We now explain some mathematical concepts related to the linear stability of difference schemes for PDEs. For simplicity, we restrict Equation (3) to one spatial dimension although the results analogously hold in three spatial dimensions. A general two-level difference scheme for Equation (3) takes the form

$$
Q \mathbf{z}_{j}^{n+1}=P \mathbf{z}_{j}^{n},
$$


where $n+1$ and $n$ denote the time level, while $j$ denotes the spatial grid position. For a network with $m$ distinct chemical species, $Q$ and $P$ are $m \times m$ matrices involving shift operators defined by $S_{ \pm} \mathbf{z}^{n}:=\left(\mathbf{z}_{j \pm 1}^{n}\right)_{j \in \mathbb{Z}}$.

Our stability analysis is performed by making use of the discrete Fourier transform defined according to $[28$, p. 264] by;

$$
\hat{\mathbf{z}}^{n}(\xi)=\frac{1}{\sqrt{2 \pi}} \sum_{j \in \mathbb{Z}} e^{-i j \xi} \mathbf{z}_{j}^{n}, \quad \text { where } i=\sqrt{-1} \text { and } \xi \in[-\pi, \pi]
$$

The discrete Fourier transforms of the shift operators is given by $\widehat{S_{ \pm} \mathbf{z}^{n}}(\xi)=e^{ \pm i \xi} \hat{\mathbf{z}}^{n}(\xi)$. Taking the discrete Fourier transform of the left- and right-hand side of Equation (4), the difference scheme can be transformed to the form $\hat{\mathbf{z}}^{n+1}=G(\xi) \hat{\mathbf{z}}^{n}$, where the quantity $G(\xi)$ is known as the amplification matrix of the difference scheme. We have the following theorem.

Theorem 3.1 ( [28, Theorem. 6.2.1]). The difference scheme (4) is stable if and only if there exist positive constants $\Delta x_{0}$ and $\Delta t_{0}$ and a non-negative constant $C$ independent of $\Delta x, \Delta t$ and $\xi$ so that

$$
\|G(\xi)\|_{2} \leq 1+C \Delta t
$$

for $0<\Delta x \leq \Delta x_{0}, 0<\Delta t \leq \Delta t_{0}$ and $\xi \in[-\pi, \pi]$.

The norm used on $G$ in Equation (5) is the matrix 2-norm defined by $\|G\|_{2}=\max _{\|\mathbf{u}\|_{2}=1}\|G \mathbf{u}\|_{2}=\sqrt{\mu_{\max }}$, where $\mu_{\max }$ is the largest eigenvalue of $G^{*} G$ and $G^{*}$ is the conjugate transpose of $G$ [34]. The spectrum of $G$, denoted by $\sigma(G)$, is the set of all eigenvalues of $G$. The spectral radius of $G$, denoted by $\rho(G)$ is defined as: $\rho(G)=\max \{|\lambda|: \lambda \in \sigma(G)\}$. From linear algebra, it is known that $\rho(G) \leq\|G\|_{2}$. Thus a necessary criterion for stability is the celebrated von Neumann condition stated in Theorem 3.2.

Theorem 3.2 ( [28, Theorem. 6.2.2]). If the difference scheme (4) is stable, then there exist positive constants $\Delta x_{0}, \Delta t_{0}$ and $C$ independent of $\Delta x, \Delta t$ and $\xi$ so that

$$
\rho(G(\xi)) \leq 1+C \Delta t
$$

for $0<\Delta x \leq \Delta x_{0}, 0<\Delta t \leq \Delta t_{0}$ and $\xi \in[-\pi, \pi]$.

In the single variable case, the von Neumann condition (6) is both a necessary and sufficient condition for stability. However, in the multivariable case, it is a necessary but not a sufficient condition for stability. This is because the relationship between spectral radius and norm is an inequality instead of an equality. However, if $G$ is a normal matrix, that is, $G^{*} G=G G^{*}$ or in particular, $G$ is Hermitian or symmetric, then $\rho(G)=\|G\|_{2}$ and the von Neumann condition is both necessary and sufficient for stability [35].

Sufficient conditions are established by making use of the next two propositions and lemma.

Proposition 3.1 ( [35, p. 84]). Suppose that the amplification matrix $G$ associated with the difference scheme (4) satisfies the von Neumann condition. Then, if $G$ is "uniformly diagonalizable", that is, for each $G$, there exists a matrix $S$ such that $S^{-1} G S=\Lambda$ is diagonal and $S$ and $S^{-1}$ are bounded independent of $\xi$ and $\Delta t$, then the scheme is stable.

Proposition 3.2 ( $\left[34\right.$, p. 368]). There is a matrix norm $\|\cdot\| \|_{s}$ such that $\|A\|_{s}=\rho(A)$ if and only if every eigenvalue of $A$ of maximum modulus is semisimple. That is, for all $\lambda \in \sigma(A)$ such that $|\lambda|=\rho(A)$, the algebraic multiplicity of $\lambda$ is equal to its geometric multiplicity. We call $\|\cdot\|_{s}$ the spectral radius norm.

Lemma 3.1. Let $B$ be an n-square matrix. Suppose that $B$ is diagonalizable such that $S^{-1} B S$ is a diagonal matrix, for some nonsingular matrix $S$. Then the matrices $S$ and $S^{-1}$ are bounded. 
Proof. Since $B$ is diagonalizable, the columns of the matrix $S$ are the linearly independent eigenvectors of $B$. Assume that the eigenvectors are normalized. The norm of any $n$-square matrix does not exceed $n$ times the absolute value of its largest element [35]. Thus $\|S\|_{2} \leq n$. Define $\triangle^{2}:=\operatorname{det}\left(S^{*} S\right)$, where $S^{*}$ is the conjugate transpose of $S$. Then $\left|\left(S^{-1}\right)_{i j}\right| \leq 1 / \triangle[35]$ and ||$\left|S^{-1}\right| \|_{2} \leq n / \triangle$. Thus the matrices $S$ and $S^{-1}$ are bounded.

We shall also make use of the following definitions in our analysis.

Definition 3.1. An $n$-square matrix $A$ is said to be semi-stable if every eigenvalue of $A$ has a nonpositive real part. It is said to be D-semi-stable if for all positive diagonal matrices $D$, the matrix $D A$ is semi-stable.

Note that if the matrix $A$ is D-semi-stable, then $I A=A$ is semi-stable, where $I$ is the identity matrix. Thus D-semi-stability implies semi-stability.

For the remainder of this paper, since we have assumed that the matrix $A$ in Equation (3) is real with finite entries, then any matrix norm of $A$ is bounded by the size of $A$ times the absolute value of its largest element [35]. Thus matrix $A$ is bounded. We shall make use of this fact. We are now in a position to analyze stability of some common difference schemes.

\section{Difference Schemes}

\subsection{The Crank-Nicolson Scheme}

The CN scheme is a second order accurate in time implicit scheme commonly employed for the time integration of initial value problems. A CN scheme for the system in Equation (3) in one spatial dimension is

$$
\frac{1}{\Delta t}\left(\mathbf{z}_{j}^{n+1}-\mathbf{z}_{j}^{n}\right)=\frac{1}{2 \Delta x^{2}} D\left[\left(\mathbf{z}_{j+1}^{n+1}-2 \mathbf{z}_{j}^{n+1}+\mathbf{z}_{j-1}^{n+1}\right)+\left(\mathbf{z}_{j+1}^{n}-2 \mathbf{z}_{j}^{n}+\mathbf{z}_{j-1}^{n}\right)\right]+\frac{1}{2} A\left(\mathbf{z}_{j}^{n+1}+\mathbf{z}_{j}^{n}\right) .
$$

Equation (7) can be rewritten as

$$
\left(I+r D-\frac{\Delta t}{2} A\right) \mathbf{z}_{j}^{n+1}-\frac{r}{2} D\left(S_{+} \mathbf{z}_{j}^{n+1}+S_{-} \mathbf{z}_{j}^{n+1}\right)=\left(I-r D+\frac{\Delta t}{2} A\right) \mathbf{z}_{j}^{n}+\frac{r}{2} D\left(S_{+} \mathbf{z}_{j}^{n}+S_{-} \mathbf{z}_{j}^{n}\right),
$$

where $r:=\frac{\Delta t}{\Delta x^{2}}$. Taking the discrete Fourier transform of Eq. (8) gives

$$
\left(I+2 r \sin ^{2}\left(\frac{\xi}{2}\right) D-\frac{\Delta t}{2} A\right) \hat{\mathbf{z}}^{n+1}=\left(I-2 r \sin ^{2}\left(\frac{\xi}{2}\right) D+\frac{\Delta t}{2} A\right) \hat{\mathbf{z}}^{n} .
$$

Thus the amplification matrix for the CN scheme is given by

$$
G=\left(I+p D-\frac{\Delta t}{2} A\right)^{-1}\left(I-p D+\frac{\Delta t}{2} A\right)
$$

where $p:=2 r \sin ^{2}\left(\frac{\xi}{2}\right) \geq 0$ for any $\xi \in[-\pi, \pi]$. Rewrite $G$ in the form

$$
G=\left(I-\frac{\Delta t}{2}(I+p D)^{-1} A\right)^{-1}(I+p D)^{-1}\left(I-p D+\frac{\Delta t}{2} A\right) .
$$

Since $D$ is a positive diagonal matrix, $(I+p D)$ and its inverse are both positive diagonal and invertible. We know that every $n$-square matrix is similar to a matrix in Jordan canonical form. Let $\lambda_{1}, \lambda_{2}, \ldots, \lambda_{k}$ be the distinct eigenvalues of $(I+p D)^{-1} A$, and let $J=\operatorname{diag}\left(J_{n_{1}}, J_{n_{2}}, \ldots, J_{n_{l}}\right), l \geq k$, be its Jordan canonical form, where each $J_{n_{i}}$ is a square Jordan block of size $n_{i}$ having eigenvalue $\lambda_{i}$ on the diagonal, 1 's on the superdiagonal and zeros elsewhere and $n_{1}+n_{2}+\cdots+n_{l}=m$, the size of $G$. There exists a nonsingular matrix $S$ such that $(I+p D)^{-1} A=S J S^{-1}$. Thus $G$ in Equation (9) can be written as

$$
G=X Y+\frac{\Delta t}{2} X H
$$


where $X:=S\left(I-\frac{\Delta t}{2} J\right)^{-1} S^{-1}, Y:=(I+p D)^{-1}(I-p D)$ and $H:=(I+p D)^{-1} A$. If $D=$ $\operatorname{diag}\left(d_{1}, d_{2}, \ldots, d_{m}\right)$, matrix $Y$ is a diagonal matrix with diagonal entries $\frac{1-p d_{j}}{1+p d_{j}}, j=1,2, \ldots, m$. We now establish sufficient conditions for the stability of the CN scheme.

Theorem 4.1. Suppose that the matrix $A$ is D-semi-stable, matrix $G$ is diagonalizable and matrix $X$ is such that every eigenvalue of maximum modulus is semi-simple. Then

(i) matrix $G$ satisfies the von Neumann condition, and

(ii) the CN scheme is unconditionally stable.

Proof. (i) Applying the spectral radius norm on Eq. (10), we have

$$
\|G\|_{s} \leq\left\|X\left|\left\|_{s}\right\| Y\left\|_{s}+\frac{\Delta t}{2}\right\| X\right|\right\|_{s}\|H\|_{s} .
$$

Since $Y$ is a diagonal matrix, Proposition 3.2 implies that $\|Y\|_{s}=\rho(Y)$. Since $p d_{j} \geq 0$, the eigenvalues of $Y$ given by $\nu_{j}=\frac{1-p d_{j}}{1+p d_{j}}$ satisfy $\left|\nu_{j}\right| \leq 1$ for all $j=1,2, \ldots, m$. Thus $\rho(Y) \leq 1$. The eigenvalues of $X$ are the same as the eigenvalues of $\left(I-\frac{\Delta t}{2} J\right)^{-1}$. If $A$ is $D$-semi-stable, all the eigenvalues of $J$, which are of course the eigenvalues of $(I+p D)^{-1} A$, have non-positive real parts. Thus, for any $\Delta t$, if $\mu$ is an eigenvalue of $\left(I-\frac{\Delta t}{2} J\right)$, then $\operatorname{Re}(\mu) \geq 1$. This implies that $|\mu| \geq 1$. Hence any eigenvalue of $\left(I-\frac{\Delta t}{2} J\right)^{-1}$, given by $1 / \mu$ will satisfy $\left|\mu^{-1}\right| \leq 1$. Thus $\rho(X) \leq 1$.

Since $G$ is diagonalizable and $X$ is such that every eigenvalue of maximum modulus is semi-simple, from Proposition 3.2, we have $\|G\|_{s}=\rho(G)$ and $\|X\|_{s}=\rho(X)$. Thus Eq. (11) reduces to $\rho(G) \leq$ $1+\Delta t \frac{1}{2}\|H\|_{s}$.

Since $(I+p D)^{-1}$ is a positive diagonal matrix for any $p \in[0,2 r]$ and matrix $A$ is bounded, matrix $H=(I+p D)^{-1} A$ is bounded. Let us denote this bound by $2 C$ for some positive constant $C$. Then $\rho(G) \leq 1+C \Delta t$.

(ii) Since $G$ is diagonalizable and satisfies the von Neumann condition in (i), the proof follows from Lemma 3.1 and Proposition 3.1.

Thus although the CN scheme has been proven to be linearly unconditionally stable for the linear diffusion equation, this cannot be established for the full RD equation unless some reasonable assumptions are made. In our case, we have proved this by making the assumption that the Jacobian matrix $A$ of the reaction part is D-semi-stable, the amplification matrix is diagonalizable and the matrix $X:=$ $\left(I-\frac{\Delta t}{2}(I+p D)^{-1} A\right)^{-1}$ is such that every eigenvalue of maximum modulus is semi-simple. D-semistability will be proved later for a large class of chemical reactions. The assumption of diagonalizability is reasonable since the set of real matrices that are diagonalizable within $\mathbf{M}_{n}(\mathbb{C})$ is dense in $\mathbf{M}_{n}(\mathbb{R})[36$, p. 87]. The assumption of the maximum absolute eigenvalue being semi-simple is even less restrictive than the diagonalizability assumption.

\subsection{The Fractional Step $\theta$-Scheme}

The fractional step $\theta$-scheme (FSTS) is an operator splitting technique introduced by Glowinski in [20] for the time integration of initial value problems. The method has also been employed in [21] to solve a system of RD equations. The scheme is applied to initial value problems of the form

$$
\mathbf{u}_{t}=\mathcal{A}(\mathbf{u}), \quad \mathbf{u}(0)=\mathbf{u}_{0},
$$

where $\mathcal{A}$ is a nonlinear operator that has a nontrivial decomposition

$$
\mathcal{A}=\mathcal{A}_{1}+\mathcal{A}_{2}
$$


To derive the FSTS, the time step is divided into three portions and over each portion, the operators $\mathcal{A}_{1}$ and $\mathcal{A}_{2}$ are alternately treated implicitly and explicitly. In the case of RD equations, it is natural to take operators $\mathcal{A}_{1}$ and $\mathcal{A}_{2}$ to be the diffusion and reaction terms, respectively. Let $\left.\theta \in\right] 0,1 / 2[$, divide the time interval $[n, n+1]$ into three sub-intervals $[n, n+\theta],[n+\theta, n+1-\theta]$ and $[n+1-\theta, n+1]$. An FSTS for Equation (3) is

$$
\left\{\begin{array}{c}
\frac{\mathbf{z}_{j}^{n+\theta}-\mathbf{z}_{j}^{n}}{\theta \Delta t}=\frac{1}{\Delta x^{2}} D\left(\mathbf{z}_{j+1}^{n+\theta}-2 \mathbf{z}_{j}^{n+\theta}+\mathbf{z}_{j-1}^{n+\theta}\right)+A \mathbf{z}_{j}^{n}, \\
\frac{\mathbf{z}_{j}^{n+1-\theta}-\mathbf{z}_{j}^{n+\theta}}{(1-2 \theta) \Delta t}=\frac{1}{\Delta x^{2}} D\left(\mathbf{z}_{j+1}^{n+\theta}-2 \mathbf{z}_{j}^{n+\theta}+\mathbf{z}_{j-1}^{n+\theta}\right)+A \mathbf{z}_{j}^{n+1-\theta}, \\
\frac{\mathbf{z}_{j}^{n+1}-\mathbf{z}_{j}^{n+1-\theta}}{\theta \Delta t}=\frac{1}{\Delta x^{2}} D\left(\mathbf{z}_{j+1}^{n+1}-2 \mathbf{z}_{j}^{n+1}+\mathbf{z}_{j-1}^{n+1}\right)+A \mathbf{z}_{j}^{n+1-\theta} .
\end{array}\right.
$$

Using an eigenvalue analysis and assuming the operator $\mathcal{A}$ in Equation (12) to be a constant $N \times N$ matrix, symmetric and positive definite, and where $\mathcal{A}_{1}, \mathcal{A}_{2}$ in Equation (13) are given by $\mathcal{A}_{1}=\alpha \mathcal{A}$, $\mathcal{A}_{2}=\beta \mathcal{A}$, with $\alpha+\beta=1,0<\alpha, \beta<1$, Glowinski [20] showed that the FSTS is second order accurate in time if $\theta=1-1 / \sqrt{2}$, otherwise the scheme is first order accurate in time. This can be confirmed for our FSTS given in Eq. (14) by multiplying the first and last equations in (14) by $\theta \Delta t$ and the second equation by $(1-2 \theta) \Delta t$, summing up the three equations and carrying out a Taylor series expansion about the point $(n+1 / 2, j)$. To proceed with a stability analysis of the FSTS in (14), rewrite the three equations in the form

$$
\left\{\begin{array}{c}
(I+2 r \theta D) \mathbf{z}_{j}^{n+\theta}-r \theta D\left(\mathbf{z}_{j+1}^{n+\theta}+\mathbf{z}_{j-1}^{n+\theta}\right)=(I+\theta \Delta t A) \mathbf{z}_{j}^{n}, \\
(I-(1-2 \theta) \Delta t A) \mathbf{z}_{j}^{n+1-\theta}=(I-2(1-2 \theta) r D) \mathbf{z}_{j}^{n+\theta}+(1-2 \theta) r D\left(\mathbf{z}_{j+1}^{n+\theta}+\mathbf{z}_{j-1}^{n+\theta}\right), \\
(I+2 r \theta D) \mathbf{z}_{j}^{n+1}-r \theta D\left(\mathbf{z}_{j+1}^{n+1}+\mathbf{z}_{j-1}^{n+1}\right)=(I+\theta \Delta t A) \mathbf{z}_{j}^{n+1-\theta},
\end{array}\right.
$$

where, as before, $r=\Delta t / \Delta x^{2}$. Taking the discrete Fourier transform of the three equations above and combining the resulting equations gives

$$
\begin{aligned}
\hat{\mathbf{z}}^{n+1}= & \left(I+4 r \theta \sin ^{2}\left(\frac{\xi}{2}\right) D\right)^{-1}(I+\theta \Delta t A)(I-(1-2 \theta) \Delta t A)^{-1} \\
& \left(I-4 r(1-2 \theta) \sin ^{2}\left(\frac{\xi}{2}\right) D\right)\left(I+4 r \theta \sin ^{2}\left(\frac{\xi}{2}\right) D\right)^{-1}(I+\theta \Delta t A) \hat{\mathbf{z}}^{n} .
\end{aligned}
$$

The amplification matrix for the FSTS is thus given by

$$
\begin{aligned}
G= & \left(I+4 r \theta \sin ^{2}\left(\frac{\xi}{2}\right) D\right)^{-1}(I+\theta \Delta t A)(I-(1-2 \theta) \Delta t A)^{-1} \\
& \left(I-4 r(1-2 \theta) \sin ^{2}\left(\frac{\xi}{2}\right) D\right)\left(I+4 r \theta \sin ^{2}\left(\frac{\xi}{2}\right) D\right)^{-1}(I+\theta \Delta t A)
\end{aligned}
$$

In the single variable case, the amplification factor takes the form

$$
G=\frac{(1+a \theta \Delta t)^{2}\left(1-4 r d(1-2 \theta) \sin ^{2}\left(\frac{\xi}{2}\right)\right)}{(1-a(1-2 \theta) \Delta t)\left(1+4 r d \theta \sin ^{2}\left(\frac{\xi}{2}\right)\right)^{2}}
$$

where $a$ is the Jacobian and $d$ the diffusion coefficient. The von Neumann condition $|G| \leq 1+C \Delta t$ is then necessary and sufficient for stability. Now,

$$
|G|=\left|\frac{1+a \theta \Delta t}{1-a(1-2 \theta) \Delta t}\right| \cdot\left|\frac{1-4 r d(1-2 \theta) \sin ^{2}\left(\frac{\xi}{2}\right)}{1+4 r d\left(2 \theta+4 r d \theta^{2} \sin ^{2}\left(\frac{\xi}{2}\right)\right) \sin ^{2}\left(\frac{\xi}{2}\right)}\right| \cdot|1+a \theta \Delta t| .
$$


Assume that the Jacobian is semi-stable such that $a \leq 0$ and let $\frac{1}{4} \leq \theta \leq \frac{1}{3}$. Then $|G| \leq|1+a \theta \Delta t| \leq$ $1+\theta|a| \Delta t \leq 1+\frac{1}{3}|a| \Delta t$. Hence, in the single variable case, if the Jacobian is semi-stable and $\theta \in[1 / 4,1 / 3]$, the FSTS is linearly unconditionally stable.

In the multivariable case where $G$ is given in Equation (15), we can only establish linear unconditional stability for the case when $\theta=\frac{1}{3}$. In this case, matrix $G$ reduces to

$$
G=P Q R\left(I+\frac{1}{3} \Delta t A\right)
$$

where $\quad P \quad:=\quad\left(I+\frac{4}{3} r \sin ^{2}\left(\frac{\xi}{2}\right) D\right)^{-1}, \quad Q \quad:=\quad\left(I+\frac{1}{3} \Delta t A\right)\left(I-\frac{1}{3} \Delta t A\right)^{-1}$ and $R:=\left(I-\frac{4}{3} r \sin ^{2}\left(\frac{\xi}{2}\right) D\right)\left(I+\frac{4}{3} r \sin ^{2}\left(\frac{\xi}{2}\right) D\right)^{-1}$. We establish in Theorem 4.2 sufficient conditions for the stability of the FSTS.

Theorem 4.2. Consider the amplification matrix $G$ given in Equation (16). Assume that $G$ is diagonalizable, matrix $A$ is semi-stable and matrix $Q$ is such that every eigenvalue of maximum modulus is semi-simple. Then

(i) G satisfies the von Neumann condition, and

(ii) the FSTS with $\theta=\frac{1}{3}$ is unconditionally stable.

Proof. (i) Taking the spectral radius norm of Equation (16), we have

$$
\|G\|_{s} \leq\|P\|\left\|_{s}\right\| Q\left\|_{s}\right\| R \|_{s}\left(1+\frac{1}{3} \Delta t\|A\|_{s}\right) .
$$

Since $G$ is diagonalizable, $P$ and $R$ are diagonal and $Q$ is such that every eigenvalue of maximum modulus is semi-simple, using Proposition 3.2, Equation (17) reduces to

$$
\rho(G) \leq \rho(P) \rho(Q) \rho(R)\left(1+\frac{1}{3}\|A\|_{s} \Delta t\right) .
$$

Since matrix $A$ is bounded, let us denote this bound by $3 C$, where $C$ is some non-negative constant. Equation (18) reduces to

$$
\rho(G) \leq \rho(P) \rho(Q) \rho(R)(1+C \Delta t) .
$$

Matrices $P$ and $R$ are both diagonal with entries and hence eigenvalues $\mu_{i}=\frac{1}{1+\frac{4}{3} r d_{i} \sin ^{2}\left(\frac{\xi}{2}\right)}$ and $\nu_{i}=\frac{1-\frac{4}{3} r d_{i} \sin ^{2}\left(\frac{\xi}{2}\right)}{1+\frac{4}{3} r d_{i} \sin ^{2}\left(\frac{\xi}{2}\right)}, i=1, \ldots, m$, respectively. Since $d_{i}>0$, it is clear that both $\left|\mu_{i}\right| \leq 1$ and $\left|\nu_{i}\right| \leq 1$. Hence

$$
\rho(P) \leq 1 \text { and } \rho(R) \leq 1
$$

To find $\rho(Q)$, let $J$ be the Jordan canonical form of $A$. There exists a nonsingular matrix $S$ such that $A=S J S^{-1}$. Matrix $Q$ can then be written as $Q=S\left(I+\frac{1}{3} \Delta t J\right)\left(I-\frac{1}{3} \Delta t J\right)^{-1} S^{-1}$. The eigenvalues of $Q$ are the same as the eigenvalues of $\left(I+\frac{1}{3} \Delta t J\right)\left(I-\frac{1}{3} \Delta t J\right)^{-1}$. Let $\lambda_{1}, \ldots, \lambda_{k}$ be the distinct eigenvalues of $A$. The matrices $\left(I+\frac{1}{3} \Delta t J\right)$ and $\left(I-\frac{1}{3} \Delta t J\right)^{-1}$ are upper triangular with diagonal entries $1+\frac{1}{3} \Delta t \lambda_{j}$ and $1-\frac{1}{3} \Delta t \lambda_{j}$, respectively. Thus matrix $\left(I+\frac{1}{3} \Delta t J\right)\left(I-\frac{1}{3} \Delta t J\right)^{-1}$ is also upper triangular with distinct 
eigenvalues $\delta_{j}=\frac{1+\frac{1}{3} \Delta t \lambda_{j}}{1-\frac{1}{3} \Delta t \lambda_{j}}, j=1, \ldots, k$. Since matrix $A$ is semi-stable, that is, $\operatorname{Re}\left(\lambda_{j}\right) \leq 0$ for all $j$, then $\left|\delta_{j}\right| \leq 1$ for all $j$. Hence

$$
\rho(Q) \leq 1
$$

Combining Equations (19) to (21) leads to $\rho(G) \leq 1+C \Delta t$.

(ii) Proceeds as in the proof for Theorem 4.1 part (ii).

\subsection{The implicit integration factor method}

The IIF method is a semi-implicit exponential integrator method due to Nie et al. in [27]. In this scheme, like in all exponential integrator methods, stiffness arising due to the discretization of the spatial diffusion term is overcome by evaluating the diffusion term exactly whereas the stiffness due to the reactions is handled by carrying out an implicit treatment of the nonlinear reaction terms. In the single variable case, this method has been proven to be linearly unconditionally stable [27].

To derive an $r$ th order accurate IIF method, Equation (2) is multiplied by an integrating factor $e^{-t \mathcal{C}}$ and then integrated over a single time step from $t=t_{n}$ to $t=t_{n+1}=t_{n}+\Delta t$ to obtain

$$
\mathbf{u}\left(t_{n+1}\right)=e^{\Delta t \mathcal{C}} \mathbf{u}\left(t_{n}\right)+e^{\Delta t \mathcal{C}} \int_{0}^{\Delta t} e^{-\tau \mathcal{C}} \mathcal{F}\left(\mathbf{u}\left(t_{n}+\tau\right)\right) \mathrm{d} \tau .
$$

The integrand, $g(\tau):=e^{-\tau \mathcal{C}} \mathcal{F}\left(\mathbf{u}\left(t_{n}+\tau\right)\right)$, is then approximated by an $(r-1)$ th degree interpolation polynomial, $P(\tau)$, using interpolation points $t_{n+1}, t_{n}, \ldots, t_{n+2-r}$ and a direct integration of the polynomial is done. Equation (22) can then be written as

$$
\mathbf{u}_{n+1}=e^{\Delta t \mathcal{C}} \mathbf{u}_{n}+e^{\Delta t \mathcal{C}} \int_{0}^{\Delta t} P(\tau) \mathrm{d} \tau
$$

For the second order IIF scheme $(r=2), P(\tau)$ is linear, and Equation $(23)$ reduces to

$$
\mathbf{u}_{n+1}=e^{\Delta t \mathcal{C}} \mathbf{u}_{n}+e^{\Delta t \mathcal{C}} \cdot \frac{\Delta t}{2}(g(0)+g(\Delta t))=e^{\Delta t \mathcal{C}}\left(\mathbf{u}_{n}+\frac{\Delta t}{2} \mathcal{F}\left(\mathbf{u}_{n}\right)\right)+\frac{\Delta t}{2} \mathcal{F}\left(\mathbf{u}_{n+1}\right) .
$$

By computing boundaries for the stability region, Nie et al. [27], proved that, in the single variable case, the second order IIF scheme is linearly unconditional stable. We use a direct approach to establish sufficient conditions for the linear stability of this scheme in the multivariable case.

Consider Equation (3) in one spatial dimension. If we discretize in space using a central difference scheme on a grid with $N$ internal grid points and assuming $\mathbf{z}=0$ at the boundary, we have

$$
\frac{\partial \mathbf{z}^{k}}{\partial t}=\frac{1}{\Delta x^{2}} D\left(\mathbf{z}^{k-1}-2 \mathbf{z}^{k}+\mathbf{z}^{k+1}\right)+A \mathbf{z}^{k}, \quad k=1,2, \ldots, N,
$$

where $\mathbf{z}^{k}=\left[z_{1}^{k}, z_{2}^{k}, \ldots, z_{m}^{k}\right]^{\top}$. Here the subscript represents the index of the chemical species whereas the superscript represents the grid point. The boundary condition implies that $\mathbf{z}^{0}=\mathbf{z}^{N+1}=0$. The linear system in Equation (25) is a system of $N m$ equations in $N m$ unknowns. Rearrange the equations in (25) so that the first $N$ equations are time derivatives of $z_{1}$ at the $N$ grid points, the next $N$ equations are for $z_{2}$, and so on. Then system (25) can be written in matrix form as

$$
\mathbf{Z}_{t}=\mathcal{C} \mathbf{Z}+\mathcal{A} \mathbf{Z}
$$

where $\mathbf{Z}=\left[z_{1}^{1}, \ldots, z_{1}^{N}, \ldots, z_{m}^{1}, \ldots, z_{m}^{N}\right]^{\top} \in \mathbb{R}^{N m}, \mathcal{C}$ is an $N m$-square block diagonal matrix arising due to diffusion and is given by

$$
\mathcal{C}=\operatorname{diag}\left(B_{1}, B_{2}, \ldots, B_{m}\right) \text { with } B_{i}=\frac{d_{i}}{\Delta x^{2}}\left(\begin{array}{cccc}
-2 & 1 & & 0 \\
1 & \ddots & \ddots & \\
& \ddots & \ddots & 1 \\
0 & & 1 & -2
\end{array}\right)_{N \times N} .
$$


$\mathcal{A}$ is a block matrix with blocks of size $N$, arising due to reaction, and is given by

$$
\mathcal{A}=\left(\begin{array}{cccc}
a_{11} I_{N} & a_{12} I_{N} & \cdots & a_{1 m} I_{N} \\
a_{21} I_{N} & a_{22} I_{N} & \cdots & a_{2 m} I_{N} \\
& \cdots & \cdots & \\
a_{m 1} I_{N} & a_{m 2} I_{N} & \cdots & a_{m m} I_{N}
\end{array}\right)=A \otimes I_{N}
$$

where $I_{N}$ is the identity matrix of size $N, a_{i j}$ is the $i j$-entry of the Jacobian matrix $A$ and $\otimes$ denotes the Kronecker product [37]. We shall make use of the following theorem.

Theorem 4.3 ( $\left[37\right.$, p. 141]). Let $A \quad \in \quad \mathbb{R}^{n \times n} \quad$ have eigenvalues $\lambda_{1}, \lambda_{2}, \ldots, \lambda_{n}$ and let $B \in \mathbb{R}^{m \times m}$ have eigenvalues $\mu_{1}, \mu_{2}, \ldots, \mu_{m}$. Then the $m n$ eigenvalues of $A \otimes B$ are $\lambda_{1} \mu_{1}, \ldots, \lambda_{1} \mu_{m}, \lambda_{2} \mu_{1}, \ldots, \lambda_{2} \mu_{m}, \ldots, \lambda_{n} \mu_{m}$

We now proceed to establish sufficient conditions for the stability of the second order IIF scheme. From Equation (24), it follows that

$$
\left(I-\frac{\Delta t}{2} \mathcal{A}\right) \mathbf{Z}_{n+1}=e^{\Delta t \mathcal{C}}\left(I+\frac{\Delta t}{2} \mathcal{A}\right) \mathbf{Z}_{n}
$$

Assume that the matrix $A$ is semi-stable. Then the matrix $I-\frac{\Delta t}{2} \mathcal{A}$ is nonsingular, and Equation (29) can be written in the form

$$
\mathbf{Z}_{n+1}=\left(I-\frac{\Delta t}{2} \mathcal{A}\right)^{-1} e^{\Delta t \mathcal{C}}\left(I+\frac{\Delta t}{2} \mathcal{A}\right) \mathbf{Z}_{n}=Q \mathbf{Z}_{n}
$$

where $Q:=\left(I-\frac{\Delta t}{2} \mathcal{A}\right)^{-1} e^{\Delta t \mathcal{C}}\left(I+\frac{\Delta t}{2} \mathcal{A}\right)$. Since the scheme in Equation (30) does not contain an amplification matrix, Proposition 3.1 used for the CN and FSTS cannot be applied to establish sufficient conditions for the stability of the second order IIF scheme. We shall instead make use of the following proposition.

Proposition 4.1 ( [28, Proposition 3.1.14]). Suppose $Q$ is similar to a symmetric matrix P, i.e. there exists a nonsingular matrix $S$ such that $Q=S P S^{-1}$. If $\|S\| \mid$ and $\left\|\left|S^{-1} \|\right|\right.$ are uniformly bounded for any matrix norm $\|\cdot\| \|$, a necessary and sufficient condition for the stability of Equation (30) is that $\rho(Q) \leq 1+\beta \Delta$ t for some non-negative $\beta$, where $\rho(Q)$ is the spectral radius of $Q$.

Define $X:=\left(I-\frac{\Delta t}{2} \mathcal{A}\right)^{-1}$ and $Y:=e^{\Delta t \mathcal{C}}$. Matrix $Q$ can be written as

$$
Q=X Y\left(I+\frac{\Delta t}{2} \mathcal{A}\right), \text { where } \mathcal{A}=A \otimes I_{N} .
$$

Sufficient conditions for the stability of the scheme are established below.

Theorem 4.4. Suppose that matrix $Q$ in Equation (31) is diagonalizable, the Jacobian matrix $A$ is semi-stable and matrix $X$ is such that every eigenvalue of maximum modulus is semi-simple. Then

(i) there exists a nonzero constant $\beta$ such that $\rho(Q) \leq 1+\beta \Delta t$, and

(ii) the second order IIF scheme $\mathbf{Z}_{n+1}=Q \mathbf{Z}_{n}$ is unconditionally stable.

Proof. (i) Applying the spectral radius norm on Eq. (31), we have

$$
\|Q\|_{s} \leq\|X\|\left\|_{s}\right\| Y \|_{s}\left(1+\frac{\Delta t}{2}\|\mathcal{A}\|_{s}\right) .
$$

Since $Q$ is diagonalizable and $X$ is such that every eigenvalue of maximum modulus is semi-simple, Proposition 3.2 implies that $\|Q\|_{s}=\rho(Q)$ and $\|X\|_{s}=\rho(X)$. To find $\rho(X)$, let $\lambda_{1}, \lambda_{2}, \ldots, \lambda_{k}$ be the 
distinct eigenvalues of the Jacobian matrix $A$. From Theorem $4.3, \lambda_{1}, \lambda_{2}, \ldots, \lambda_{k}$ will also be the distinct eigenvalues of $\mathcal{A}=A \otimes I_{N}$. Let $J$ be the Jordan canonical form of $\mathcal{A}$. There exists a nonsingular matrix $S$ such that $\mathcal{A}=S J S^{-1}$. Matrix $X$ can then be written as $X=S\left(I-\frac{\Delta t}{2} J\right)^{-1} S^{-1}$. The eigenvalues of $X$ are the same as the eigenvalues of $\left(I-\frac{\Delta t}{2} J\right)^{-1}$. Since matrix $A$ is semi-stable, the distinct eigenvalues of $\left(I-\frac{\Delta t}{2} J\right)$, given by $1-\frac{\Delta t}{2} \lambda_{1}, 1-\frac{\Delta t}{2} \lambda_{2}, \ldots, 1-\frac{\Delta t}{2} \lambda_{k}$, have positive real parts greater than or equal to 1 . Hence $\left|1-\frac{\Delta t}{2} \lambda_{i}\right| \geq 1$ for all $i$ and for any $\Delta t$. Therefore all the distinct eigenvalues of $\left(I-\frac{\Delta t}{2} J\right)^{-1}$, which are in fact the eigenvalues of $X$, and given by $\left(1-\frac{\Delta t}{2} \lambda_{1}\right)^{-1},\left(1-\frac{\Delta t}{2} \lambda_{2}\right)^{-1}, \ldots,\left(1-\frac{\Delta t}{2} \lambda_{k}\right)^{-1}$, have modulus less than or equal to 1 . Thus

$$
\rho(X) \leq 1 .
$$

Matrix $\Delta t \mathcal{C}$ is real and block diagonal given by Equation (27) with each $B_{i}$ multiplied by $\Delta t$. Thus matrix $Y=e^{\Delta t \mathcal{C}}$ is a real block diagonal matrix given by $Y=\operatorname{diag}\left(e^{\Delta t B_{1}}, e^{\Delta t B_{2}}, \ldots, e^{\Delta t B_{m}}\right)$. Since each of the matrices $B_{j}$ is real symmetric, each of the matrices $e^{\Delta t B_{j}}$ is also real symmetric and therefore $Y$ is a real symmetric block diagonal matrix. We know from linear algebra that every real symmetric matrix is diagonalizable. This implies that every eigenvalue of $Y$ is semi-simple and thus from Proposition 3.2, $\|Y\|_{s}=\rho(Y)$. The spectrum of a block diagonal matrix is the union of the spectra of the diagonal blocks [38]. Thus the set of eigenvalues of $Y$ is the union of the sets of eigenvalues of the $e^{\Delta t B_{j}}, j=1, \ldots, m$. Note that the matrices $\Delta t B_{j}$ are symmetric tridiagonal and thus can be diagonalized. That is, there exists nonsingular $E_{j}$ such that $\Delta t B_{j}=E_{j} \Lambda_{j} E_{j}^{-1}$, where $\Lambda_{j}$ is diagonal with diagonal entries being the eigenvalues of $\Delta t B_{j}$. Thus

$$
e^{\Delta t B_{j}}=E_{j} e^{\Lambda_{j}} E_{j}^{-1} \text { and } e^{\Lambda_{j}}=\operatorname{diag}\left(e^{\mu_{1}}, e^{\mu_{2}}, \ldots, e^{\mu_{N}}\right),
$$

where $\mu_{k}$ is an eigenvalue of $\Delta t B_{j}, k=1, \ldots, N$. The eigenvalues of $\Delta t B_{j}$ are given by $\mu_{k}=-2 r d_{j}+$ $2 r d_{j} \cos \left(\theta_{k}\right)=-4 r d_{j} \sin ^{2}\left(\frac{\theta_{k}}{2}\right)$ where $\theta_{k}=\frac{k \pi}{N+1}$ and $r=\frac{\Delta t}{\Delta x^{2}}$. Thus $0<e^{\mu_{k}}=e^{-4 r d_{j} \sin ^{2}\left(\frac{\theta_{k}}{2}\right)} \leq 1$ for $k=1, \ldots, N$. Hence

$$
\rho\left(e^{\Delta t B_{j}}\right) \leq 1 \text { for } j=1, \ldots, m, \text { which implies } \rho(Y) \leq 1 .
$$

Combining Equations (32) to (34), we have $\rho(Q) \leq 1+\frac{\Delta t}{2}\|\mathcal{A}\|_{s}$.

Since matrix $\mathcal{A}$ is a constant matrix of size $N m,\|\mathcal{A}\|_{s}$ is bounded by $N m$ times the absolute value of its largest element [35]. Let us denote this bound by $2 \beta$. Thus we have $\rho(Q) \leq 1+\beta \Delta t$.

(ii) Since $Q$ is diagonalizable and satisfies part (i) above, the proof follows from Lemma 3.1 and Proposition 4.1 .

\subsection{Implicit-explicit (IMEX) methods}

For problems in which stiffness is only due to diffusion and not the reactions, it is reasonable to use implicit-explicit methods. In these methods, the stiff linear diffusion is treated implicitly, while the (nonlinear) reaction terms are treated explicitly. This has the advantage that the resulting algebraic system is linear and can thus be solved without the need for nonlinear iterative solvers which makes the computation cheaper. For details on IMEX methods, we refer to [23,24].

An IMEX scheme for Equation (3) in one spatial dimension is

$$
\frac{1}{\Delta t}\left(\mathbf{z}_{j}^{n+1}-\mathbf{z}_{j}^{n}\right)=\frac{1}{\Delta x^{2}} D\left(\mathbf{z}_{j+1}^{n+1}-2 \mathbf{z}_{j}^{n+1}+\mathbf{z}_{j-1}^{n+1}\right)+A \mathbf{z}_{j}^{n}
$$

Equation (35) can be written as

$$
(I+2 r D) \mathbf{z}_{j}^{n+1}-r D\left(\mathbf{z}_{j+1}^{n+1}+\mathbf{z}_{j-1}^{n+1}\right)=(I+\Delta t A) \mathbf{z}_{j}^{n},
$$


where as before $r:=\Delta t / \Delta x^{2}$. Taking the discrete Fourier transform gives

$$
\left(I+4 r D \sin ^{2} \frac{\xi}{2}\right) \hat{\mathbf{z}}^{n+1}=(I+\Delta t A) \hat{\mathbf{z}}^{n}
$$

The amplification matrix for the IMEX scheme is given by

$$
G=(I+p D)^{-1}(I+\Delta t A),
$$

where $p:=4 r \sin ^{2} \frac{\xi}{2}, \xi \in[-\pi, \pi]$. Sufficient conditions for the stability of the IMEX scheme are established in Theorem 4.5 .

Theorem 4.5. Suppose that matrix $G$ in (37) is diagonalizable. Then

(i) G satisfies the von Neumann condition, and

(ii) the IMEX scheme in Equation (35) is unconditionally stable.

Proof. (i) Applying the spectral radius norm to Equation (37), we have

$$
\|G \mid\|_{s} \leq\left\|(I+p D)^{-1}\right\|_{s}\|I+\Delta t A\|_{s} .
$$

Since matrix $G$ is diagonalizable, $\|G\|_{s}=\rho(G)$. Matrix $(I+p D)^{-1}$ is diagonal with diagonal entries $1 /\left(1+p d_{i}\right)$ and $\left\|(I+p D)^{-1}\right\|_{s}=\rho\left((I+p D)^{-1}\right)$. Thus

$$
\rho(G) \leq \rho\left((I+p D)^{-1}\right) \cdot\|I+\Delta t A\|_{s} .
$$

The eigenvalues of $(I+p D)^{-1}$ are its diagonal entries $1 /\left(1+p d_{i}\right)$ and each of them is less than 1 since $p, d_{i} \geq 0$. Thus $\rho\left((I+p D)^{-1}\right) \leq 1$. Equation (38) reduces to

$$
\rho(G) \leq\|I+\Delta t A\|_{s} \leq\|I\|_{s}+\Delta t\|A\|_{s}=1+\Delta t\|A\|_{s} .
$$

Since matrix $A$ is bounded, G satisfies the von Neumann condition.

(ii) Proceeds as in the proof of Theorem 4.1 part (ii).

\section{Matrix semi-stability}

In Section 4, we have established sufficient conditions for the linear stability of some well-known difference schemes used for the time integration of the multi-variable RD equation (1). One notable condition has been that the Jacobian $A$ of the reaction part is either D-semi-stable or semi-stable. We demonstrate here that for a single chemical reaction, by making reasonable assumptions on the kinetics of the reaction, the Jacobian $A$ evaluated at any $\mathbf{u} \in \mathbb{R}_{+}^{n}$, is indeed both semi-stable and D-semi-stable. We also show that the assumptions that we make in order to prove the semi-stability of $A$ hold for reactions governed by a variety of enzyme-kinetic rate governing laws.

\subsection{Monotone kinetics}

Here we give a brief description about the assumption that we make on the kinetics of the reaction. Consider a reversible chemical reaction involving $n$ chemical species $X_{1}, X_{2}, \ldots, X_{n}$ that takes the form:

$$
\sum_{i=1}^{n} \alpha_{i} X_{i} \underset{v_{r}}{\stackrel{v_{f}}{\rightleftharpoons}} \sum_{i=1}^{n} \beta_{i} X_{i},
$$

where $\alpha_{i}$ and $\beta_{i}$ are nonnegative integers, known as the stoichiometric coefficients of species $X_{i}$ on the substrate and product sides of the equation, respectively and $v_{f}$ and $v_{r}$ are the reaction rates in the forward and backward directions, respectively. Denote by $x_{i}$ the concentration of the chemical species $X_{i}$ and let $x=\left[x_{1}, \ldots, x_{n}\right]^{\top} \in \mathbb{R}_{+}^{n}$ denote the vector of species concentrations at any time $t$, where the superscript $T$ denotes transpose. Assume that reaction (39) satisfies the following properties: 
(a) The reaction is non-autocatalytic, that is, no chemical species appears on both the left (substrates) and right (products) side of the reaction. This means that for each $i$, either $\alpha_{i}=0$ or $\beta_{i}=0$.

(b) The forward and backward reaction rates are continuously differentiable functions of the species concentrations $x$. Thus the overall reaction rate $v$ in the forward direction, given by $v=v_{f}-v_{r}$, is a continuously differentiable function of species concentrations. That is $v=v(x)$.

(c) Define $\gamma_{i}:=\beta_{i}-\alpha_{i}$. For each $X_{i}$, if $\gamma_{i}<0$ then $\frac{\partial v(x)}{\partial x_{i}} \geq 0$, while if $\gamma_{i}>0$ then $\frac{\partial v(x)}{\partial x_{i}} \leq 0$.

All reactions whose reaction rates satisfy properties (b) and (c) above are said to obey monotone kinetics [39].

We now proceed to show that the Jacobian of the reaction part of an RD system modelling the dynamics of a non-autocatalytic reaction with monotone kinetics is D-semi-stable.

\subsection{D-semi-stability}

The concept of D-stability is commonly encountered in mathematical economics and there are many necessary and sufficient conditions for a matrix to be D-stable, although many of them are hard to prove [40]. One notable property about D-stable matrices, which we have already shown in Section 3, is that every D-stable matrix is also a stable matrix although the converse is not generally true [40]. Thus in order for us to show that a matrix $A$ is both semi-stable and D-semi-stable, we only need to show that $A$ is D-semi-stable and use this to infer the semi-stability of $A$.

Suppose the chemical reaction (39) is non-autocatalytic. Let us order the chemical species in such a way that the first $m$ chemical species, $X_{1}, \ldots, X_{m}$, appear as reactants, while the remaining $(n-m)$ species, $X_{m+1}, \ldots, X_{n}$, appear as products. Then Equation (39) can be written as;

$$
\sum_{i=1}^{m} \alpha_{i} X_{i} \underset{v_{r}}{\stackrel{v_{f}}{\rightleftharpoons}} \sum_{j=m+1}^{n} \beta_{j} X_{j} .
$$

The stoichiometric matrix $N$ for reaction (40) is given by

$$
N=\left[-\alpha_{1} \cdots-\alpha_{m} \beta_{m+1} \cdots \beta_{n}\right]^{\top} .
$$

The time evolution of the concentrations of the chemical species in reaction (40) is governed by the RD system

$$
\frac{\partial x}{\partial t}=D \nabla^{2} x+N v(x)
$$

where $v(x)$ is the overall reaction rate. D-semi-stability of the Jacobian for the reaction part is established in the following theorem.

Theorem 5.1. Consider the non-autocatalytic reaction (40) with stoichiometric matrix $N$ given in Equation (41) and overall reaction rate $v(x)$. Suppose the reaction kinetics are monotone. Then the Jacobian matrix $A$ of $f(x):=N v(x)$, evaluated at any $x \in \mathbb{R}_{+}^{n}$, is D-semi-stable.

Proof. Since $N$ is a column vector, denote by $n_{k}$ the $k$ th-entry of $N$. The Jacobian $A$ of $f(x)$ is the $n$-square matrix $A=\left[a_{i j}\right]$ with $a_{i j}=n_{i} \frac{\partial v(x)}{\partial x_{j}}$, for $i, j=1, \ldots, n$. Denote by $D$ a positive diagonal matrix given by $D=\operatorname{diag}\left(\delta_{1}, \delta_{2}, \ldots, \delta_{n}\right)$. Matrix $D A$ is given by

$$
D A=\left[\begin{array}{cccccc}
-\delta_{1} \alpha_{1} \frac{\partial v(x)}{\partial x_{1}} & \cdots & -\delta_{1} \alpha_{1} \frac{\partial v(x)}{\partial x_{m}} & -\delta_{1} \alpha_{1} \frac{\partial v(x)}{\partial x_{m}+1} & \cdots & -\delta_{1} \alpha_{1} \frac{\partial v(x)}{\partial x_{n}} \\
& \ddots & & & & \\
-\delta_{m} \alpha_{m} \frac{\partial v(x)}{\partial x_{1}} & \cdots & -\delta_{m} \alpha_{m} \frac{\partial v(x)}{\partial x_{m}} & -\delta_{m} \alpha_{m} \frac{\partial v(x)}{\partial x_{m+1}} & \cdots & -\delta_{m} \alpha_{m} \frac{\partial v(x)}{\partial x_{n}} \\
\delta_{m+1} \beta_{m+1} \frac{\partial v(x)}{\partial x_{1}} & \cdots & \delta_{m+1} \beta_{m+1} \frac{\partial v(x)}{\partial x_{m}} & \delta_{m+1} \beta_{m+1} \frac{\partial v(x)}{\partial x_{m+1}} & \cdots & \delta_{m+1} \beta_{m+1} \frac{\partial v(x)}{\partial x_{n}} \\
\delta_{n} \beta_{n} \frac{\partial v(x)}{\partial x_{1}} & \cdots & \delta_{n} \beta_{n} \frac{\partial v(x)}{\partial x_{m}} & \delta_{n} \beta_{n} \frac{\partial v(x)}{\partial x_{m+1}} & \cdots & \delta_{n} \beta_{n} \frac{\partial v(x)}{\partial x_{n}}
\end{array}\right]
$$


Our aim is to show that matrix $D A$ is semi-stable.

Note that the matrix $D A$ has rank 1. From linear algebra, it is known that the number of nonzero eigenvalues of a matrix is less than or equal to the rank of the matrix. Therefore matrix $D A$ has at most one nonzero eigenvalue and thus zero is an eigenvalue of $D A$ with algebraic multiplicity of at least $n-1$. Since matrix $D A$ is supposed to have $n$ eigenvalues counting multiplicity, the remaining one eigenvalue of $D A$ is therefore real and is given by the trace of $D A$. Let us denote this eigenvalue by $\lambda$. Thus

$$
\lambda=\sum_{i=1}^{m}-\alpha_{i} \delta_{i} \frac{\partial v(x)}{\partial x_{i}}+\sum_{j=m+1}^{n} \beta_{j} \delta_{j} \frac{\partial v(x)}{\partial x_{j}} .
$$

Since we have monotone kinetics, $\frac{\partial v(x)}{\partial x_{i}} \geq 0$ for $i=1, \ldots, m$ and $\frac{\partial v(x)}{\partial x_{j}} \leq 0$ for $j=m+1, \ldots, n$. Thus each term in both the first and second summations in (43) are nonpositive and hence both sums are nonpositive. Therefore $\lambda \leq 0$ and the matrix $D A$ is semi-stable. This proves that the matrix $A$ is D-semi-stable.

From the properties of D-stable matrices, we conclude that matrix $A$ is also semi-stable.

We now demonstrate on some examples that the assumption we made about the kinetics of the reaction being monotone is a reasonable assumption that is satisfied by many rate governing laws.

Example 5.1. Consider the reversible chemical reaction governed by mass-action kinetics: $\alpha_{1} X_{1}+\alpha_{2} X_{2}+$ $\cdots+\alpha_{m} X_{m} \underset{k_{-1}}{\stackrel{k_{1}}{\rightleftharpoons}} \beta_{m+1} X_{m+1}+\cdots+\beta_{n} X_{n}$.

Here, $k_{1}$ and $k_{-1}$ are positive rate constants. Denote by $x_{i}$ the concentration of species $X_{i}$. The overall rate of reaction $v$ in the forward direction is given by $v(x)=k_{1} \prod_{i=1}^{m} x_{i}^{\alpha_{i}}-k_{-1} \prod_{i=m+1}^{n} x_{i}^{\beta_{i}}$. For $i=1, \ldots, m$, $\gamma_{i}=-\alpha_{i}<0$ and $\frac{\partial v(x)}{\partial x_{i}}=k_{1} \alpha_{i} x_{i}^{\alpha_{i}-1} \prod_{\substack{j=1 \\ j \neq i}}^{m} x_{j}^{\alpha_{j}}>0$, since $x \in \mathbb{R}_{+}^{n}$. Similarly, for $i=m+1, \ldots, n$, $\gamma_{i}=\beta_{i}>0$ and $\frac{\partial v(x)}{\partial x_{i}}=-k_{-1} \beta_{i} x_{i}^{\beta_{i}-1} \prod_{\substack{j=m+1 \\ j \neq i}}^{n} x_{j}^{\beta_{j}}<0$. Thus the reaction rate $v(x)$ satisfies the properties of monotone kinetics.

Example 5.2. Consider the reversible enzymatic reaction: $\quad S \stackrel{E}{\rightleftharpoons} P$. Here $S$ is the substrate, $E$ is the enzyme that acts as a catalyst for the reaction and $P$ the product. Let $s$ and $p$ denote the substrate and product concentrations, respectively, at any time $t$, and $x=\left[\begin{array}{ll}s p\end{array}\right]^{\top} \in \mathbb{R}_{+}^{2}$. Note that $\gamma_{s}=-1$ while $\gamma_{p}=1$. Suppose the reaction is governed by Michaelis-Menten kinetics, then

$$
v(x)=\frac{\frac{V_{f}}{K s} s-\frac{V_{r}}{K_{p}} p}{1+\frac{s}{K_{s}}+\frac{p}{K_{p}}},
$$

where $V_{f}, V_{r}, K_{s}$ and $K_{r}$ are positive constants. It can be verified that $\frac{\partial v(x)}{\partial s}>0$ and $\frac{\partial v(x)}{\partial p}<0$. Thus the reaction has monotone kinetics.

\section{Numerical simulations of the Brusselator model}

In this section we test our results by carrying out a simulation of the Brusselator RD system. The Brusselator model (see e.g. [41-43]) is a well-known theoretical model for the study of dissipative structures in nonlinear chemical systems and also in the study of biological pattern formation. It describes 
the space-time dependence of the concentrations of the intermediate products $U$ and $V$ in the sequence of reactions

$$
A \stackrel{k_{1}}{\longrightarrow} U, B+U \stackrel{k_{2}}{\longrightarrow} V+D, 2 U+V \stackrel{k_{3}}{\longrightarrow} 3 U, U \stackrel{k_{4}}{\longrightarrow} E .
$$

Assuming that all rate constants of the reactions are unity and the concentrations of $\mathrm{A}$ and $\mathrm{B}$ are constants, the conventional dimensionless Brusselator model in a one-dimensional domain can be written as

$$
\left\{\begin{array}{l}
\frac{\partial u}{\partial t}=D_{u} \frac{\partial^{2} u}{\partial x^{2}}+u^{2} v+a-(b+1) u \\
\frac{\partial v}{\partial t}=D_{v} \frac{\partial^{2} v}{\partial x^{2}}-u^{2} v+b u
\end{array},(x, t) \in[0, L] \times[0, \infty),\right.
$$

where $D_{u}$ and $D_{v}$ are the diffusion coefficients of $U$ and $V$, respectively. We complete the model system by imposing homogeneous Neumann boundary conditions

$$
u_{x}(0, t)=u_{x}(L, t)=0 \text { and } v_{x}(0, t)=v_{x}(L, t)=0,
$$

and nonnegative initial conditions

$$
u(x, 0)=u_{0}(x) \geq 0, \quad v(x, 0)=v_{0}(x) \geq 0 .
$$

There are no known analytical solutions to the Brusselator RD system and thus it has to be solved numerically. The system has a unique homogeneous equilibrium solution at $u=a, v=b / a$. In the absence of diffusion, this equilibrium solution is stable when the parameters $(a, b)$ satisfy

$$
b<a^{2}+1 .
$$

In order for the system to exhibit spatial patterns, Turing's diffusion-driven instability requires the homogeneous equilibrium to be unstable in the presence of diffusion. This is achieved when

$$
b>\left(1+a \sqrt{\frac{D_{u}}{D_{v}}}\right)^{2} .
$$

Simulation results

The schemes described in this work are used to simulate the Brusselator system for $x \in[0,10]$ and taking the initial condition to be a small perturbation of the homogeneous equilibrium solution. The simulations are carried out for two cases; one case where the Jacobian of the reaction part is semi-stable at every time step of the simulation and another case in which it is not semi-stable. The results from the simulations are compared to those generated by an explicit scheme (forward in time, centered in space).

\section{Case I: Jacobian is semi-stable}

The parameter values chosen are $D_{u}=1, D_{v}=8, a=4.5, b=9$ and $\theta=1 / 3$ for the FSTS. The results are shown in Figures 1 and 2.

\section{Case II: Jacobian is not semi-stable}

Here all the parameter values stay the same as above except $b$ which we set to 21 . Setting $\Delta t=0.01$, the solutions generated by the CN, FSTS, IIF and IMEX all converge to the solution generated by the explicit scheme as shown in Figures 3 and 4 . However, setting $\Delta t=0.255$ for the CN scheme, $\Delta t=0.2$ for the FSTS and $\Delta t=0.25$ for the IIF leads to a blow-up of the solutions. This is shown in Figure 5 . In fact all these schemes blow up for $\Delta t$ bigger than a certain value. 

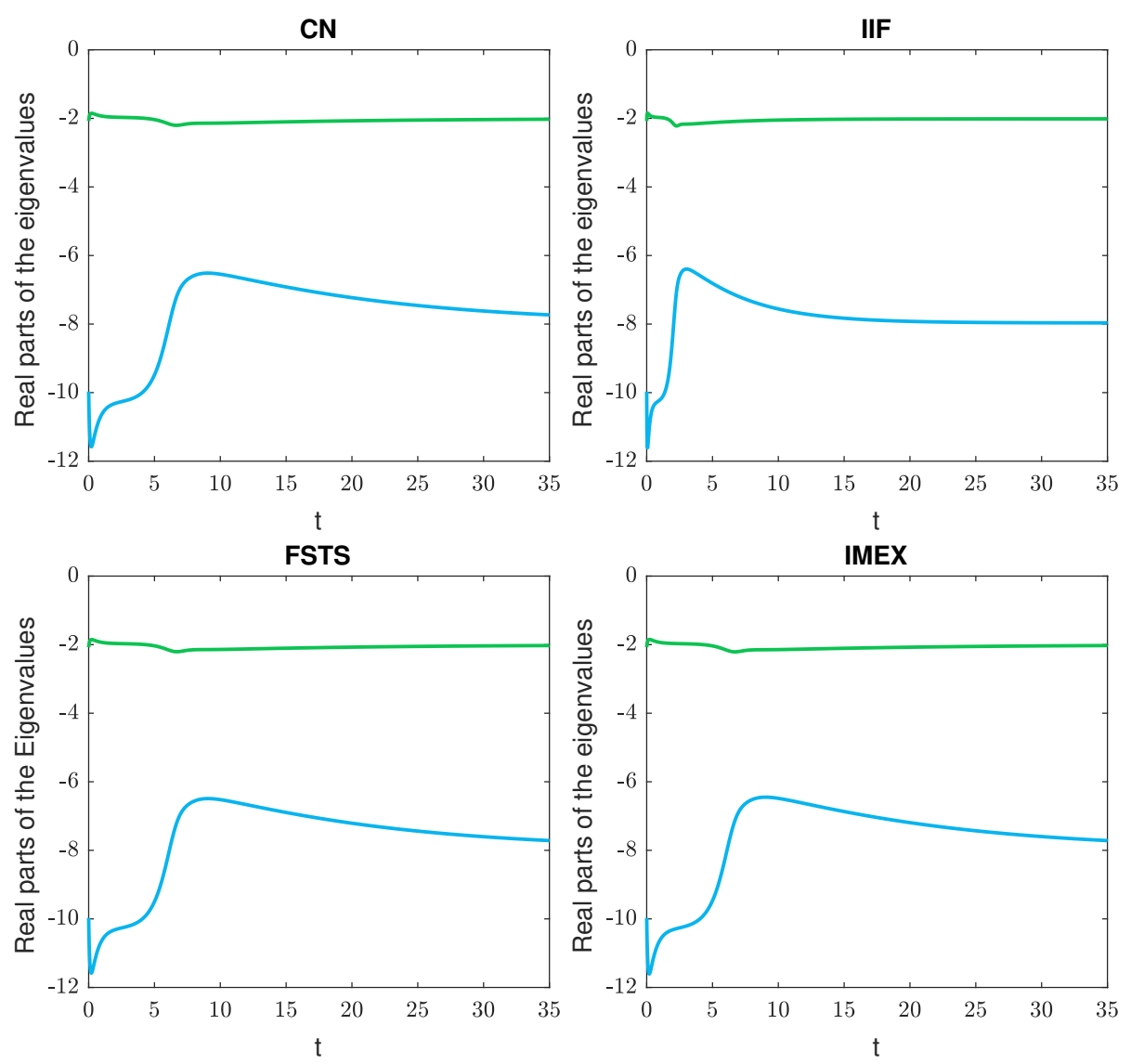

Figure 1. Real parts of the two eigenalues of the Jacobian at the point $x=5.05$. The space step size is $\Delta x=0.101$, while time step is $\Delta t=0.0002$ for the explicit scheme and $\Delta t=0.01$ for the rest of the schemes.
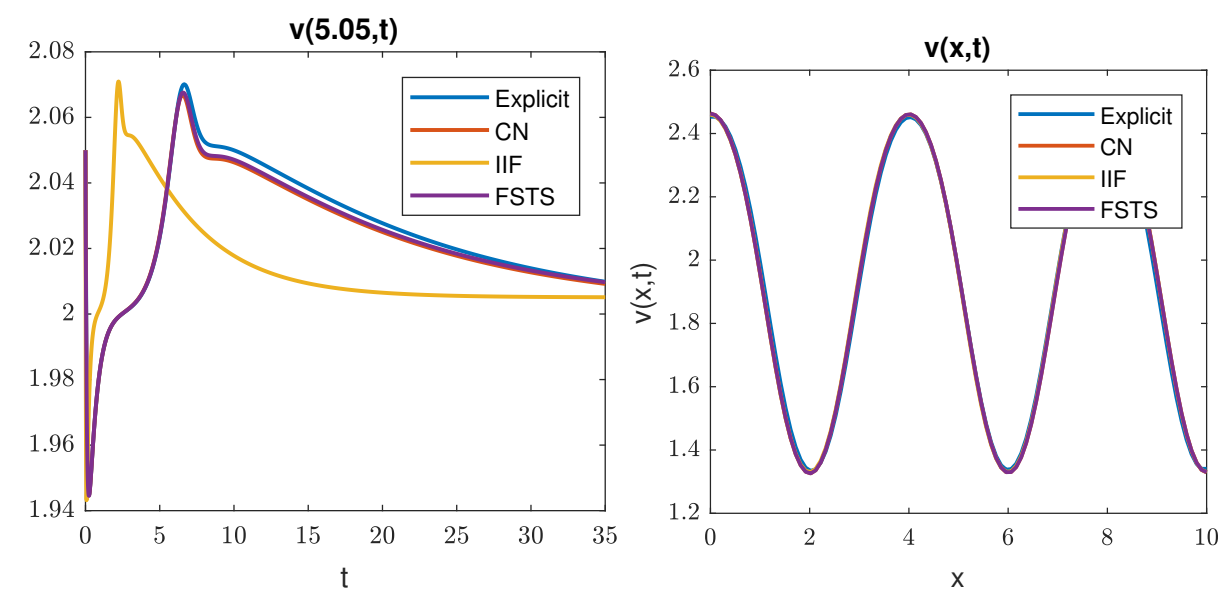

Figure 2. Left: Solution $v(x, t)$ of the Brusselator system at $x=5.05$. The space step size is $\Delta x=0.101$, while time step is $\Delta t=0.0002$ for the explicit scheme and $\Delta t=0.01$ for the rest of the schemes. Right: Spatial pattern solution.

\section{Discussion and Conclusion}

In this work, we have established sufficient conditions for the local linear stability of some commonly applied implicit finite difference schemes used for the solution of the multivariable RD equation (1). In particular, we have derived conditions for the fully implicit CN and FSTS, the semi-implicit IIF and IMEX schemes. It should, however, be noted that the conditions derived for the stability of localized linear system may not guarantee stability of schemes for the nonlinear system (1). But it is known that 

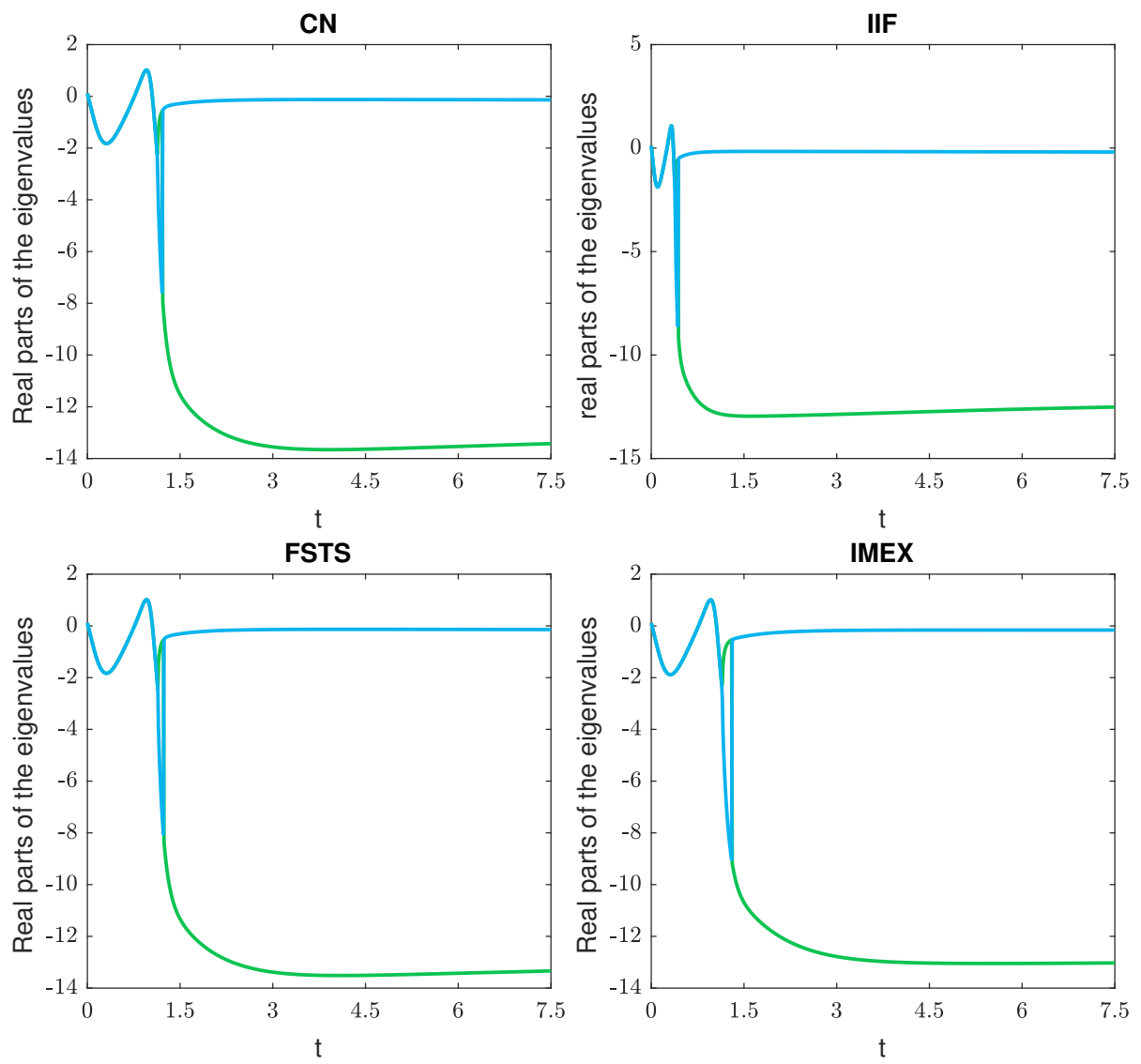

Figure 3. Real parts of the two eigenalues of the Jacobian for $D_{u}=1, D_{v}=8, a=4.5, b=21$ at the point $x=5.05$. The space step size is $\Delta x=0.101$, while time step is $\Delta t=0.0002$ for the explicit scheme and $\Delta t=0.01$ for the rest of the schemes.
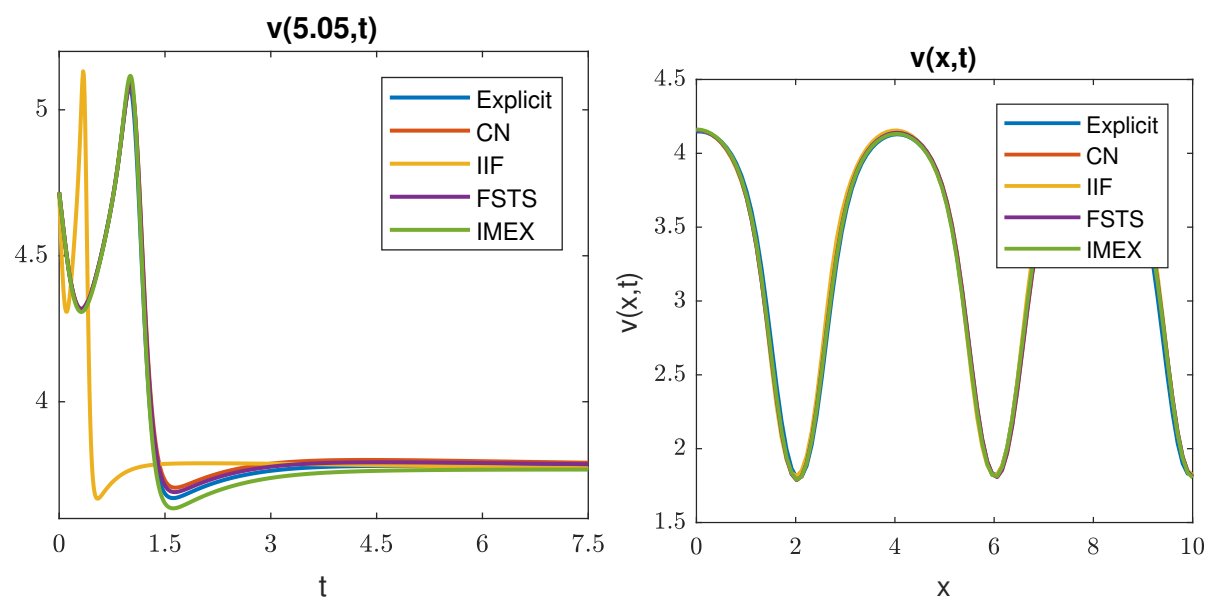

Figure 4. Left: Solution $v(x, t)$ for $D_{u}=1, D_{v}=8, a=4.5, b=21$ at the point $x=5.05$ with $\Delta t=0.01$. Right: Spatial pattern.

for schemes of the nonlinear system to be stable, the same schemes should be stable for the localized linear version of the nonlinear system.

One notable sufficient condition is that the Jacobian matrix of the reaction part evaluated at any known positive solution is D-semi-stable, for the case of the CN scheme and semi-stable for the case of the FSTS and IIF schemes. We have showed in Section 5 that the assumption of semi-stability and Dsemi-stability of the Jacobian matrix is reasonable and is satisfied by a large class of chemical reactions, 

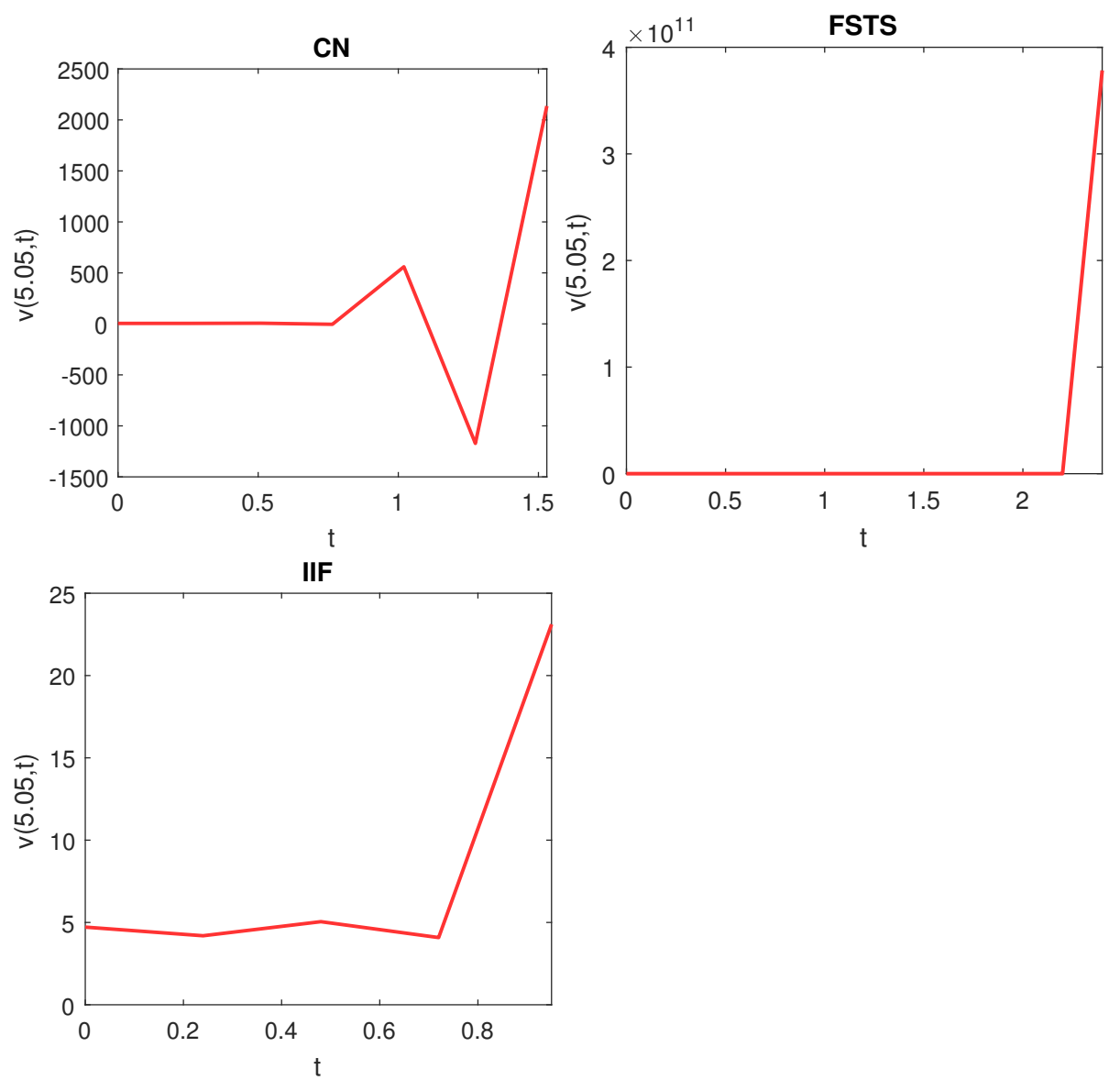

Figure 5. Blow-up of solution $v(5.05, t)$ for $\Delta t=0.255$ for the CN, $\Delta t=0.2$ for the FSTS and $\Delta t=0.25$ for the IIF. One of the eigenvalues of the Jacobian is positive at the time of blow-up.

namely, those that obey monotone kinetics.

For the FSTS, our method has only been able to establish stability for $\theta=\frac{1}{3}$. However, the FSTS scheme is first order accurate in time at this value of $\theta$. In contrast, both the CN and IIF schemes are second order accurate in time.

Figure 2 shows that the solution generated by the IIF scheme is not as close to the explicit scheme solution as all the other schemes. The reason may be that the IIF scheme was designed for diffusiondominated problems and does not seem to work well for problems where the reactions dominate. However, the scheme converges to the same spatial pattern which is usually the main interest of RD applications in biology. Our analysis also establishes unconditional stability of the IMEX scheme by only making the assumption of diagonalizability of the amplification matrix.

The main results of this paper are the Theorems 4.1, 4.2, 4.4 and 4.5 used in establishing sufficient conditions for the stability of the CN, FSTS, IIF and IMEX schemes, respectively. The proofs of these theorems are similar in nature. All the theorems except Theorem 4.4 are based on the amplification matrix arising out of the use of the discrete Fourier transform. Theorem 4.4 is based on the matrix arising out of a direct manipulation of the scheme.

We hope that the stability analysis that we have carried out in this paper will help us in deriving an efficient difference scheme that is unconditionally stable, second order accurate in time and also easier to implement than the implicit schemes considered in this paper. We also intend to show that the Dsemi-stability that has been proven for a single non-autocatalytic reaction with monotone kinetics can be extended to a network of chemical reactions. 


\section{References}

1. R. Fisher, The wave of advance of advantageous genes, Ann Hum Genet, vol. 7, pp. 355-369, 1937.

2. A. D. Bazykin, Hypothetical mechanism of speciation, Evolution, vol. 23, pp. 685-687, 1969.

3. B. Bradshaw-Hajek, Reaction-diffusion equations for population genetics. PhD thesis, School of Mathematics and Applied Statistics, University of Wollongong, 2004.

4. J. G. Skellam, Random dispersal in theoretical populations, Biometrika, vol. 38, pp. 196-218, 1951.

5. A. M. Turing, The chemical basis of morphogenesis, Phil. Trans. R. Soc. Lond., pp. 37-72, 1952.

6. A. Gierer and H. Meinhardt, A theory of biological pattern formation, kybernetik, vol. 12, pp. 30-39, 1972.

7. J. D. Murray, Mathematical Biology: I. An Introduction. Springer, 1989.

8. J. D. Murray, Mathematical Biology: II. Spatial Models and Biomedical Applications. Springer, 2003.

9. J. J. Tyson and P. C. Fife, Target patterns in a realistic model of the belousov-zhabotinskii reaction, J. Chem. Phys., vol. 73, pp. 2224-2237, 1980.

10. J. Sneyd, A. C. Charles, and M. J. Sanderson, A model for the propagation of intracellular calcium waves, Am. J. Physiol., vol. 266, pp. 293-302, 1994.

11. J. Sneyd, B. T. R. Wetton, A. C. Charles, and M. J. Sanderson, Intracellular calcium waves mediated by diffusion of inositol triphosphate; a two dimensional model, Am. J. Physiol., vol. 268, pp. 1537$1545,1995$.

12. S. Means, A. J. Smith, J. Shepherd, J. Shadid, J. Fowler, R. J. H. Wojcikiewicz, T. Mazel, G. D. Smith, and B. S. Wilson, Reaction-diffusion modelling of calcium dynamics with realistic er geometry, Biophysical Journal, vol. 91, pp. 537-557, 2006.

13. X.-S. Yang, Computational modelling of nonlinear calcium waves, Appl. Math. Model., vol. 30, pp. 200-208, 2006.

14. M. A. Colman, C. Pinali, A. W. Trafford, H. Zhang, and A. Kitmitto, A computational model of spatiotemporal cardiac intracellular calcium handling with realistic structure and spatial flux distribution from sarcoplasmic reticulum and t-tubule reconstructions, PLOS Comput. Biol., vol. 13, 2017.

15. E. Meron, Pattern formation in excitable media, Physics Reports, vol. 218, no. 1, pp. 1 - 66, 1992.

16. J. M. Hyman, The method of lines solution of partial differential equations, Tech. Rep. C00-3077-139, New York University, October 1976.

17. L. Brugnano, F. Mazzia, and D. Trigiante, Fifty years of stiffness, in Recent Advances in Computational and Applied Mathematics (T. E. Simos, ed.), ch. 1, pp. 1-21, Springer Netherlands, 2011.

18. K. Radhakrishnan, Comparison of numerical techniques for integration of stiff ordinary differential equations arising in combustion chemistry, Tech. Rep. NASA-TP-2372, NASA, October 1984.

19. J. Crank and P. Nicolson, A practical method for numerical evaluation of solutions of partial differential equations of the heat-conduction type, Adv. Comput. Math., vol. 6, pp. 207-226, 1996.

20. R. Glowinski, Finite element methods for incompressible viscous flow, Handbook of Numerical Analysis, vol. 9, pp. 3-1176, 2003.

21. A. Madzvamuse and A. H. Chung, Fully implicit time-stepping schemes and non-linear solvers for systems of reaction diffusion equations, Appl. Math. Comput., vol. 244, pp. 361-374, 2014.

22. A.-K. Kassam and L. N. Trefethen, Fourth-order time-stepping for stiff pdes, SIAM J. Sci. Comput., vol. 26, no. 4, pp. 1214-1233, 2005.

23. U. M. Ascher, S. J. Ruuth, and B. T. R. Wetton, Implicit-explicit methods for time-dependent partial differential equations, SIAM J. Numer. Anal., vol. 32, no. 3, pp. 797-823, 1995.

24. H. Wang, C.-W. Shu, and Q. Zhang, Stability and error estimates of local discontinuous galerkin methods with implicit-explicit time-marching for advection-diffusion problems, SIAM J. Numer. Anal., vol. 53, pp. 206-227, 2015.

25. A. Madzvamuse, Time-stepping schemes for moving grid finite elements applied to reaction-diffusion systems on fixed and growing domains, J. Comput. Phys., vol. 214, pp. 239-263, 2006. 
26. Q. Nie, Y.-T. Wan, Frederic Y. M.and Zhang, and X.-F. Liu, Compact integration factor methods in high spatial dimensions, J. Comput. Phys., vol. 227, pp. 5238-5255, 2008.

27. Q. Nie, Y.-T. Zhang, and Z. Rui, Efficient semi-implicit schemes for stiff systems, J. Comput. Phys., vol. 214, pp. 521-537, 2006.

28. J. W. Thomas, Numerical Partial Differential Equations: Finite Difference Methods. Springer, 1995.

29. D. Hoff, Stability and convergence of finite difference methods for systems of nonlinear reactiondiffusion equations, SIAM J. Numer. Anal., vol. 15, no. 6, pp. 1161-1177, 1978.

30. A. Araújo, S. Barbeiro, and P. Serranho, Stability of finite difference schemes for complex diffusion processes, SIAM J. Numer. Anal., vol. 50, no. 3, pp. 1284-1296, 2012.

31. A. Araújo, S. Barbeiro, and P. Serranho, Stability of finite difference schemes for nonlinear complex reaction-diffusion processes, IMA J. Numer. Anal., vol. 35, pp. 1381-1401, 2015.

32. N. Li, J. Steiner, and S. Tang, Convergence and stability analysis of an explicit finite difference method for 2-dimensional reaction-diffusion equations, J. Austral. Math. Soc., vol. 36, pp. 234-241, 1994.

33. C. Hirsch, Numerical Computation of Internal and External Flows: Fundamentals of Numerical Discretization. John Wiley \& Sons, 1988.

34. R. A. Horn and C. R. Johnson, Matrix Analysis. Cambridge, 2013.

35. R. D. Richtmyer and K. W. Morton, Difference Methods for Initial-Value Problems. John Wiley \& Sons, 1967.

36. D. Serre, Matrices; Theory and Applications. Springer, 2010.

37. A. J. Laub, Matrix Analysis for Scientists and Engineers. SIAM, 2005.

38. B. Dasgupta, Applied Mathematical Methods. Dorling Kindersley (India) Pvt. Ltd., 2006.

39. D. Siegel, Chemical reaction networks as compartmental systems, Presented at the 21st International Symposium on Mathematical Theory of Networks and Systems, July 7-11, 2014. Groningen, The Netherlands.

40. G. Giorgi and C. Zuccotti, An overview on d-stable matrices. Universita Di Pavia, DEM Working Paper Series, Feb. 2015.

41. G. Nicolis and I. Prigogine, Self-organization in nonequilibrium systems : from dissipative structures to order through fluctuations / g. nicolis, i. prigogine, 011977.

42. I. Prigogine and R. Lefever, Symmetry breaking instabilities in dissipative systems. ii, The Journal of Chemical Physics, vol. 48, no. 4, pp. 1695-1700, 1968.

43. M. Marek and I. Schreiber, Chaotic behaviour of deterministic dissipative systems. Cambridge University Press, 1991. 